\title{
All-in-one relaxion: A unified solution to five particle-physics puzzles
}

\author{
R. S. Gupta, J. Y. Reiness, and M. Spannowsky \\ Institute for Particle Physics Phenomenology, Durham University, \\ South Road, Durham DH1 3LE, United Kingdom
}

(Received 24 May 2019; published 5 September 2019)

\begin{abstract}
We present a unified relaxion solution to the five major outstanding issues in particle physics: Higgs mass naturalness, dark matter, matter-antimatter asymmetry, neutrino masses and the strong $C P$ problem. The only additional field content in our construction with respect to standard relaxion models is an up-type vectorlike fermion pair and three right-handed neutrinos charged under the relaxion shift symmetry. The observed dark matter abundance is generated automatically by oscillations of the relaxion field that begin once it is misaligned from its original stopping point after reheating. The matter-antimatter asymmetry arises from spontaneous baryogenesis induced by the $C P T$ violation due to the rolling of the relaxion after reheating. The $C P T$ violation is communicated to the baryons and leptons via an operator, $\partial_{\mu} \phi J^{\mu}$, where $J^{\mu}$ consists of right-handed neutrino currents arising naturally from a simple neutrino mass model. Finally, the strong $C P$ problem is solved via the Nelson-Barr mechanism, i.e., by imposing $C P$ as a symmetry of the Lagrangian that is broken only spontaneously by the relaxion. The $C P$ breaking is such that although an $\mathcal{O}(1)$ strong Cabibbo-Kobayashi-Maskawa (CKM) phase is generated, the induced strong $C P$ phase is much smaller, i.e., within experimental bounds.
\end{abstract}

DOI: $10.1103 /$ PhysRevD.100.055003

\section{INTRODUCTION}

Recently, particle physics research has been driven to a large extent by the expectation of physics beyond the Standard Model (BSM) at the TeV scale. While there are many theoretical and observational reasons to extend the Standard Model (SM) — such as Higgs mass naturalness, dark matter, matter-antimatter asymmetry, neutrino masses and the strong $C P$ problem-only the first of these issues necessarily requires $\mathrm{TeV}$-scale new physics. In fact, if Higgs mass naturalness is ignored and new physics scales far beyond the $\mathrm{TeV}$ scale are allowed, the other issues can be solved by very minimal extensions of the SM [1-6].

It is arguably far more challenging to find an explanation (apart from tuning or anthropics) for a light Higgs mass with a high new physics scale. While conventional wisdom says this is impossible, the recently proposed cosmological relaxation (or relaxion) models [7] aim to find just such an explanation. In these models the rolling of the so-called relaxion field during inflation leads to a scanning of the Higgs mass squared from positive to negative values. Once the Higgs mass squared becomes negative it triggers a backreaction potential that stops the scanning soon after, at a value much smaller than the new physics scale.

Published by the American Physical Society under the terms of the Creative Commons Attribution 4.0 International license. Further distribution of this work must maintain attribution to the author(s) and the published article's title, journal citation, and DOI. Funded by SCOAP ${ }^{3}$.
We show in this paper that the relaxion construction has many interesting built-in features that can provide solutions to multiple other BSM puzzles in a way that is completely different from the other examples referred to above. These features are: spontaneous $C P T$ violation during its rolling, spontaneous $C P$ violation when it stops and oscillations about its stopping point after reheating. The spontaneous $C P T$ violation leads to spontaneous baryogenesis during the rolling of the relaxion after reheating [8]; the spontaneous $C P$ violation leads to a Nelson-Barr solution $[9,10]$ of the strong $C P$ problem [11,12]; and the relaxion oscillations generate the observed dark matter abundance [13]. The spontaneous baryogenesis mechanism requires that baryons and/or leptons are charged under the relaxion shift symmetry. In this work the relaxion shift symmetry is identified with a Froggatt-Nielsen symmetry [14], under which three new right-handed $(\mathrm{RH})$ neutrino states (but no SM states) are charged. This satisfies the requirement of spontaneous baryogenesis while also giving an explanation for the smallness of neutrino masses.

Thus, we achieve a unified solution to five BSM puzzles, namely the lightness of the Higgs boson in the absence of $\mathrm{TeV}$ scale new physics, dark matter, matter-antimatter asymmetry, neutrino masses and the strong $C P$ problem.

\section{REVIEW AND BASIC SETUP}

In relaxion models, the Higgs mass squared parameter is promoted to a dynamical quantity $\mu^{2}(\phi)$, which varies due to its couplings to the relaxion field, $\phi$, 


$$
V_{\text {roll }}=\mu^{2}(\phi) H^{\dagger} H+\lambda_{H}\left(H^{\dagger} H\right)^{2}-r_{\text {roll }}^{2} M^{4} \cos \frac{\phi}{F},
$$

with,

$$
\mu^{2}(\phi)=\kappa M^{2}-M^{2} \cos \frac{\phi}{F} .
$$

Here, $H$ is the SM Higgs doublet, $\lambda_{H}$ is its quartic coupling, $M$ is the UV cutoff of the Higgs effective theory and $\kappa \lesssim 1$ [15]. The rolling starts from a relaxion field value, $\phi<\phi_{c}=-\left|F \cos ^{-1} \kappa\right|$, such that $\mu^{2}>0$. After crossing the point $\phi=\phi_{c}, \mu^{2}$ becomes negative, prompting electroweak symmetry breaking. This in turn activates the backreaction potential, which induces periodic "wiggles" on top of the linear envelope,

$$
V_{\mathrm{br}}=\Lambda_{c}^{4} \cos \frac{\phi}{f_{k}} .
$$

Here, $\Lambda_{c}^{4}=m^{n} v(\phi)^{4-n}$, is an increasing function of the Higgs vacuum expectation value (VEV). These wiggles cause the relaxion field to come to a halt soon after, generating a large hierarchy between the Higgs VEV and the cutoff $M$. As discussed in [7], the cutoff, $M$, cannot be raised to an arbitrarily high value because of cosmological requirements,

$$
M \lesssim\left(\frac{M_{P}}{r_{\text {roll }}}\right)^{1 / 2}\left(\frac{\Lambda_{c}^{4}}{f_{k}}\right)^{1 / 6}
$$

The relaxion mechanism must be complemented by a new mechanism at the scale $M$ (for e.g., supersymmetry [16] or Higgs compositeness [17]) to solve the full hierarchy problem up to the Planck scale.

Let us now discuss what happens after inflation. For the backreaction sector we adopt the non-QCD model of [7], where $\phi$ is the axion of a new strong sector. If the reheating temperature is greater than the critical temperature of the chiral phase transition of the new sector, i.e., if $T_{\mathrm{r}}>T_{\mathrm{c}} \sim \sqrt{4 \pi} f_{\pi^{\prime}}$, the wiggles disappear and the relaxion starts rolling again. Here $f_{\pi^{\prime}}$ is the "pion decay constant" of the new sector. When the universe cools below $T_{\mathrm{c}}$ again, the backreaction potential reappears and the rolling eventually stops provided, $m_{\phi} \lesssim 5 H\left(T_{\mathrm{c}}\right)$. This condition is obtained by demanding that the relaxion does not have enough kinetic energy to overshoot the barriers once the backreaction potential reappears $[13,18,19]$. If satisfied, the relaxion enters a slow-roll-like regime with,

$$
V^{\prime}(\phi)=5 H \dot{\phi} .
$$

It is this second phase of rolling that can lead to a generation of both the observed dark matter abundance as well as the baryon asymmetry. The explanation for dark matter requires no additional ingredient. This is due to the fact that during the second phase of rolling, the relaxion gets misaligned from its original stopping point by an angle [13],

$$
\Delta \theta=\frac{\Delta \phi}{f} \simeq \frac{1}{20}\left(\frac{m_{\phi}}{H\left(T_{\mathrm{c}}\right)}\right)^{2} \tan \frac{\phi_{0}}{f} .
$$

As shown in Ref. [13], this sets off relaxion oscillations that can give rise to the observed dark matter relic abundance,

$$
\Omega h^{2} \simeq 3 \Delta \theta^{2}\left(\frac{\Lambda_{d}}{1 \mathrm{GeV}}\right)^{4}\left(\frac{100 \mathrm{GeV}}{T_{\mathrm{osc}}}\right)^{3} .
$$

Note that the correct relic density can always be reproduced by choosing an appropriate value of $\tan \frac{\phi_{0}}{f}$. While there is some room for this in the relaxion mechanism, as the relaxion is spread across multiple vacua at the end of its rolling, the probability distribution of the relaxion field peaks for $\mathcal{O}(1)$ values of $\tan \frac{\phi_{0}}{f}$ [20]. Thus the extent to which $\tan \frac{\phi_{0}}{f}$ deviates from unity can be interpreted as a measure of the tuning required to get the correct relic abundance.

It was shown in [8] that with just one additional ingredient, this second phase of rolling can also give spontaneous relaxion baryogenesis (SRB). One requires that some fermions with $B+L$ charge are charged under the relaxion shift symmetry. This leads to the presence of the operator, $\partial_{\mu} \phi J^{\mu} / f$, where $J^{\mu}$ contains the $B+L$ current. This operator generates a chemical potential for $B+L$ violation once the second phase of relaxion rolling results in a $C P T$-breaking expectation value for $\partial_{\mu} \phi$. A baryon asymmetry is consequentially generated via $(B+L)$-violating sphaleron transitions.

As shown later, generation of the observed baryon asymmetry requires a hierarchy $f \ll f_{k}$. This and the fact that the relaxion, in any case, requires a large hierarchy between $f_{k}$ and its field excursion during rolling, $f_{k} \ll F$, are problematic as explained in [21]. The solution to generating the latter hierarchy is embedding the relaxion construction in a so-called clockwork model [22-24]; this can easily be extended to also generate the former hierarchy, giving $f \ll f_{k} \ll F$. In clockwork models there is a system of interacting complex scalars, $\Phi_{i}$, all of which get a VEV such that $\left\langle\Phi_{i}\right\rangle=\frac{f}{\sqrt{2}} e^{i \pi_{i} / f}$. There is an approximate Abelian symmetry, $\mathrm{U}(1)_{i}$, at each site which is spontaneously broken to give rise to a corresponding pseudoGoldstone mode $\pi_{i}$. Explicit breaking effects give the angular fields, $\pi_{i}$, a mass matrix such that the lightest state is a massless (Goldstone) mode given by,

$$
\phi \propto \sum_{j} \frac{\pi_{j}}{3^{j}}=\pi_{0}+\frac{\pi_{1}}{3}+\cdots+\frac{\pi_{N}}{3^{N}} .
$$




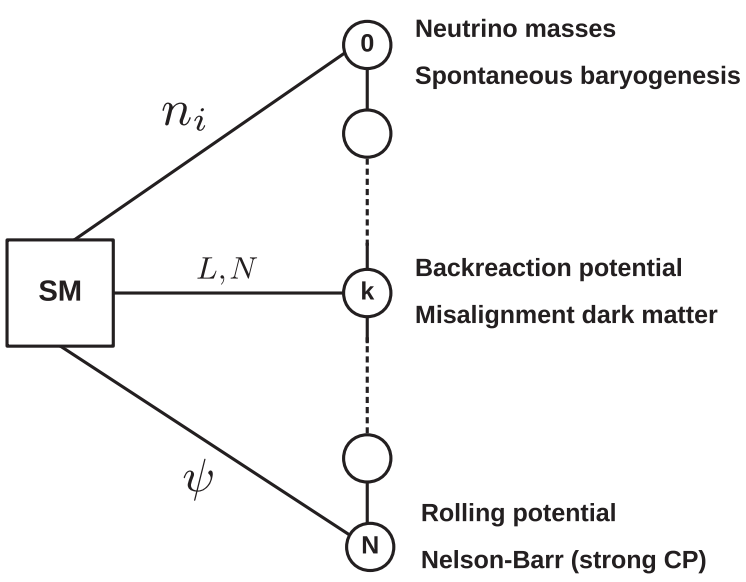

FIG. 1. Schematic representation of our set-up with the vertical line representing the clockwork system. The three right-handed neutrinos at the 0th site, $n_{i}$, couple to the SM in the usual way, generating neutrino masses; they also provide the current in the all-important operator for spontaneous baryogenesis, $\mathcal{O}_{\mathrm{SB}}=\partial_{\mu} \phi J^{\mu}$. At the $k$ th site we introduce a new strong sector which couples to SM via its fermionic matter content, $\left(L, L^{c}, N, N^{c}\right)$. This sector generates the backreaction wiggles and relaxion oscillations inside these wiggles generate the observed dark matter abundance. Finally, at site $N$ there is a Nelson-Barr sector that radiatively generates the rolling potential while also providing a solution to the strong $C P$ problem. This sector couples to the SM up sector via a new vector-like quark pair $\left(\psi, \psi^{c}\right)$.

Given that the mixing angle $\left\langle\pi_{k} \mid \phi\right\rangle \sim 3^{-k}$, any Lagrangian term where the angular field $\pi_{k}$ couples with a decay constant $f$ translates to an interaction of $\phi$ with an exponentially enhanced effective decay constant, $3^{k} f$, in the mass basis. Coming back to our setup, the hierarchy $f \ll f_{k} \ll F$ can be obtained by having the operator, $\mathcal{O}_{\mathrm{SB}}$, at the 0 -th site, the backreaction sector at the intermediate $k$ th site and the rolling potential at the $N$ th site, such that $f_{k}=3^{k} f$ and $F=3^{N} f$. This is schematically shown in Fig. 1. The rolling and backreaction potentials eventually lift the flat direction in Eq. (8), giving a mass to the relaxion.

The SRB setup reviewed above is incomplete in two respects: the operator, $\mathcal{O}_{\mathrm{SB}}$, and the rolling potential are nonrenormalizable and introduced in a somewhat ad hoc way. While $\mathcal{O}_{\mathrm{SB}}$ arises naturally if baryons and/or lepton are charged under the Abelian symmetry of which the relaxion is a Goldstone boson, charging the SM fermions seems to have no purpose other than generating $\mathcal{O}_{\mathrm{SB}}$. Furthermore, the charge assignments have to be carefully chosen such that they are anomalyfree with respect to QCD (to avoid generating a strong $C P$ phase) and preferably also with respect to electromagnetism (to avoid the generation of a $\phi \gamma \gamma$ coupling that rules out most of the parameter space in this set-up [8]). Here we complete the SRB set-up as follows: (i) Instead of introducing the operator, $\mathcal{O}_{\mathrm{SB}}$, by hand, we propose a simple neutrino mass model at site 0 , which generates this operator with a current containing only three new right-handed $(\mathrm{RH})$ neutrino fields. The operator arises because the RH fields are charged under the relaxion shift symmetry, which in turn is identified with a Froggatt-Nielsen symmetry. This also explains the observed smallness of neutrino masses. Only the SM-singlet RH neutrinos are charged under the relaxion shift symmetry, which is thus automatically anomaly-free with respect to both QCD and electromagnetism.

(ii) We show that the rolling potential can be generated by the addition of a single up-type vector-like pair at site $N$. This modification can also solve the strong $C P$ problem via the Nelson-Barr mechanism $[9,10]$. In Nelson-Barr models, $C P$ is a good symmetry in the UV and is spontaneously broken at an intermediate scale to generate an $\mathcal{O}(1)$ Cabibbo-Kobayashi-Maskawa (CKM) phase but a much smaller strong $C P$ phase (within allowed constraints). We borrow the Nelson-Barr relaxion sector from [11], where the relaxion phase upon stopping results in an $\mathcal{O}(1)$ CKM phase.

Also, spontaneous baryogenesis is an attractive potential feature from the point of view of the Nelson-Barr relaxion model, as it does not require explicit $C P$ violation.

\section{NEUTRINO MASSES AND SPONTANEOUS BARYOGENESIS}

\section{A. Getting the operator $\mathcal{O}_{\text {SB }}$}

At site 0 we introduce a sector that simultaneously generates small neutrino masses and an operator suitable for spontaneous baryogenesis. We introduce three RH neutrinos, $n_{i}$, that are charged under the 0th site Abelian symmetry, $\mathrm{U}(1)_{0}$. We fix the charge of $\Phi_{0}$ to be -1 under this symmetry and take all SM fields to be neutral. The Lagrangian for the couplings of these $\mathrm{RH}$ neutrinos is given by,

$\mathcal{L}_{\mathrm{FN}} \supset y_{n}^{i j}\left(\frac{\Phi_{0}}{\Lambda_{F N}}\right)^{q_{n_{j}}} l_{i}^{\dagger} H n_{j}+\left(\frac{\Phi_{0}}{\Lambda_{F N}}\right)^{q_{n_{i}}+q_{n_{j}}} \hat{M}_{n}^{i j} n_{i} n_{j}$,

where $l_{i}^{\dagger}$ are right handed spinors denoting the SM lepton doublets, $q_{n_{j}}$ are the Abelian charges for the sterile neutrinos and $M_{n}^{i j}$ is the Majorana mass matrix. Given that we will eventually use the Nelson-Barr solution to the strong $C P$ problem, we impose $C P$ as an exact symmetry of the Lagrangian so that all couplings above are real. Substituting, $\left\langle\Phi_{0}\right\rangle=\frac{f}{\sqrt{2}} e^{i \pi_{0} / f}$, we obtain exponentiallysuppressed effective Yukawa couplings and Majorana masses, $\quad Y_{n}^{i j}=y_{n}^{i j}\left(\epsilon_{F N}\right)^{q_{n_{j}}}$ and $\quad M_{n}^{i j}=\hat{M}_{n}^{i j}\left(\epsilon_{F N}\right)^{q_{n_{i}}+q_{n_{j}}}$, where $\epsilon_{F N}=f / \sqrt{2} \Lambda_{F N}<1$. 
Factors of $e^{i \pi_{0} / f}$, that appear upon substitution of $\Phi_{0}$ in Eq. (9), can be rotated away by the field redefinition $n_{j} \rightarrow n_{j} e^{-i \pi_{0} q_{n_{j}} / f}$, which, through the redefinition of the kinetic terms for the $\mathrm{RH}$ neutrinos, yields the desired operator, $\mathcal{O}_{\mathrm{SB}}$,

$$
\frac{q_{n_{i}}}{f}\left(\partial_{\mu} \pi_{0}\right) n_{i}^{\dagger} \bar{\sigma}^{\mu} n_{i} \rightarrow \frac{q_{n_{i}}}{f}\left(\partial_{\mu} \phi\right) n_{i}^{\dagger} \bar{\sigma}^{\mu} n_{i},
$$

where we ignore an $\mathcal{O}(1)$ factor corresponding to $\left\langle\pi_{0} \mid \phi\right\rangle$.

\section{B. Getting baryon asymmetry from $\mathcal{O}_{\mathrm{SB}}$}

The presence of $\mathcal{O}_{\mathrm{SB}}$ can lead to spontaneous baryogenesis, an idea developed in $[25,26]$. The essential feature of this mechanism is the presence of a rolling field that breaks $C P T$, a role played here by the relaxion [8].

During the second phase of the rolling, the operator $\mathcal{O}_{\mathrm{SB}}$ causes equal and opposite shifts in the energies of particles versus antiparticles, implying,

$$
\mu_{i}=-\bar{\mu}_{i}=q_{i} \dot{\phi} / f+\left(B_{i}-L_{i}\right) \mu_{B-L}+Q_{i} \mu_{Q}+T_{3 i} \mu_{T_{3}},
$$

where $\mu_{i}\left(\bar{\mu}_{i}\right)$ is the chemical potential for (anti-) particles of the $i$ th specie; $q_{i}$ is its charges under $\mathrm{U}(1)_{0}$ (which is nonzero only for the RH neutrinos); $Q_{i}$ is the electromagnetic charge; $T_{3 i}$ is the charge corresponding to the diagonal generator of $\mathrm{SU}(2)_{L}$; and the chemical potentials $\mu_{Q, T_{3}, B-L}$ have been introduced to enforce conservation of $Q, T_{3}$, and $B-L$. In the presence of $(B+L)$-violating sphaleron processes, we find,

$$
\begin{aligned}
n_{i}-\bar{n}_{i} & =f(T, \mu)-f(T, \bar{\mu}) \\
& =g_{i} \mu_{i} \frac{T^{2}}{6}, \quad g_{i} \mu_{i} \frac{T^{2}}{3},
\end{aligned}
$$

for fermions and bosons respectively, where $f(T, \mu)$ is the Fermi-Dirac (Bose-Einstein) distribution for fermions (bosons). We have taken $\mu \ll T$ and the factor $g_{i}$ denotes the number of degrees of freedom for each species. The quantities $\mu_{T_{3}, Q, B-L}$ can be obtained by imposing $n_{T_{3}}=n_{Q}=n_{B-L}=0$. For temperatures above the critical temperature for the electroweak phase transition, we obtain the following chemical potentials,

$\mu_{Q}=-\frac{3}{14} \frac{Q_{n} \dot{\phi}}{f} \quad \mu_{T_{3}}=\frac{3}{14} \frac{Q_{n} \dot{\phi}}{f} \quad \mu_{B_{L}}=\frac{33}{112} \frac{Q_{n} \dot{\phi}}{f}$,

taking all the $q_{n_{i}}=Q_{n}$. We subsequently obtain a baryon number density,

$$
n_{B}=-n_{L}=g_{\mathrm{SB}} \frac{\dot{\phi}}{f} \frac{T^{2}}{6},
$$

where $g_{\mathrm{SB}}=3 \quad Q_{n} / 4$ and finally for its ratio with the entropy density, $\eta \equiv \frac{n_{B}}{s}=g_{\mathrm{SB}} \frac{\dot{\phi}}{f} \frac{T^{2}}{6} \times\left(\frac{2 \pi^{2} g_{*} T^{3}}{45}\right)^{-1}=\frac{15}{4 \pi^{2}} \frac{g_{\mathrm{SB}}}{g_{*}} \frac{\dot{\phi}}{f T}$.

The equilibrium distribution changes after electroweak symmetry breaking, when there is no longer a need to conserve $T_{3}$. This gives $\mu_{Q}=-(4 / 11) \mu_{B-L}=-Q_{n} \dot{\phi} / 12 f$ and, once again, $g_{\mathrm{SB}}=3 Q_{n} / 4$. However, species such as the RH fermions, which are coupled very weakly to the thermal plasma, would not be able to reequilibrate on the timescale of the electroweak phase transition. The precise value of $g_{\mathrm{SB}}$ is thus hard to compute without considering the full dynamics of the process and may be different from the value obtained above. Given that these subtleties only lead to an $\mathcal{O}(1)$ ambiguity in $g_{\mathrm{SB}}$, for definiteness we stick to the value derived in Eq. (13).

The value of $\eta$ is frozen at $T=T_{\mathrm{sph}}=130 \mathrm{GeV}$, the temperature at which the sphaleron processes decouple [27]. Demanding the observed baryon asymmetry, $\eta_{0}=8.7 \times 10^{-11}$, we obtain,

$$
\frac{f_{k}}{f}=\sqrt{\frac{2}{5}} \frac{2 \pi^{3} g_{*}^{3 / 2} T_{\mathrm{sph}}^{3} \eta_{0}}{9 g_{\mathrm{SB}} m_{\phi}^{2} M_{\mathrm{Pl}}} \sim 10^{9}\left(\frac{m_{\phi}}{10^{-5} \mathrm{eV}}\right)^{-2},
$$

using $V^{\prime}=5 H \dot{\phi} \sim \Lambda_{c}^{4} / f_{k}$ (see Sec. II). It is crucial that the relaxion keeps rolling with a nonzero $\dot{\phi}$ when the value of $\eta$ is frozen at $T=T_{\mathrm{sph}}$. To ensure this, we require that the critical temperature for the phase transition of the strong sector, $T_{\mathrm{c}}$, is lower than $T_{\mathrm{sph}}$.

We have assumed so far that the $\mathrm{RH}$ neutrinos are relativistic and in equilibrium at the temperatures relevant to the calculation. The first condition requires that the seesaw scale, $M_{n} \lesssim T_{\text {sph }}$, where $M_{n}^{i j} \sim M_{n}$. The second requirement implies that the interaction rate of $n_{i}$ with SM particles satisfies, $\Gamma(n)>H\left(T_{\text {sph }}\right)$. Taking, $\Gamma(n) \sim g^{2} Y_{N}^{2} T$ [28], where $g$ is the weak coupling and $Y_{n}^{i j} \sim Y_{n}$ and requiring $\Gamma>H\left(T_{\text {sph }}\right)$, we get $Y_{n} \gtrsim 10^{-8}$.

\section{Neutrino masses}

The Lagrangian in Eq. (9) generates masses for the SM neutrinos $m_{\nu} \sim Y_{n}^{2} v^{2} / M_{n} \lesssim 0.1 \mathrm{eV}$. Given that spontaneous baryogenesis demands $M_{n} \lesssim T_{\mathrm{sph}}$, we require small effective Yukawas $Y_{n} \lesssim 10^{-6}$, which can be naturally obtained via the Froggatt-Nielsen mechanism, as explained above.

The constraints derived in the previous subsections imply that our model requires a finite range for both the effective Yukawa coupling and effective Majorana mass scale: $10^{-8} \lesssim Y_{n} \lesssim 10^{-6}, 30 \mathrm{MeV} \lesssim M_{n} \lesssim T_{\text {sph }}$. Note that sterile neutrinos with masses below $500 \mathrm{MeV}$ are in tension with big-bang nucleosynthesis (BBN) [29]. Masses around a few $\mathrm{GeV}$ may be within reach of future experiments such as SHiP [29]. For $\epsilon_{\mathrm{FN}}=0.1$ the above range of the Yukawa couplings can be obtained for $6 \leq Q_{n} \leq 8$, where we have 
taken all $q_{n_{i}}=Q_{n}$. Given that all the couplings in Eq. (9) are real, a concrete prediction of our model is a $C P$-even neutrino mass matrix, which is still comfortably allowed by neutrino experiments [30].

\section{NELSON-BARR SECTOR AND THE ROLLING POTENTIAL}

\section{A. Generating the rolling potential}

As shown in [11], the rolling potential in Eq. (1), (2) can be generated by a minimal modification of the SM up sector, namely the addition of a vectorlike pair, $\left(\psi, \psi^{c}\right)$, where $\psi$ has the same quantum numbers as an up-type singlet. Furthermore, as shown in [31], the same modification can also give a Nelson-Barr solution $[9,10]$ to the strong $C P$ problem, provided we impose an additional $Z_{2}$ symmetry. The Lagrangian terms for the relevant interactions are,

$$
\begin{aligned}
\mathcal{L}_{\mathrm{NB}}= & Y_{i j}^{u} Q \tilde{H} u^{c}+y_{\psi}^{i} \psi \Phi_{N} u_{i}^{c}+\tilde{y}_{\psi}^{i} \psi \Phi_{N}^{*} u_{i}^{c} \\
& +\mu_{\psi} \psi \psi^{c}+\text { H.c. }
\end{aligned}
$$

The $\psi, \psi^{c}$, and $\Phi_{N}$ are odd under the $Z_{2}$ symmetry, which forbids the term $Q H \psi^{c}$. Recall that an exact $C P$ symmetry has been imposed and thus all couplings are real. The $\mathrm{U}(1)_{N}$ symmetry is collectively broken by $y_{\psi}^{i}$ and $\tilde{y}_{\psi}^{i}$, leading to breaking of the relaxion shift symmetry and radiative generation of the rolling potential in Eq. (1), (2) with,

$$
\begin{gathered}
M \sim \frac{\sqrt{y_{\psi}^{i} \tilde{y}_{\psi}^{j}\left(Y^{u^{\dagger}} Y^{u}\right)_{i j}}}{4 \pi} f, \\
r_{\text {roll }} \sim \frac{4 \pi \sqrt{y_{\psi}^{k} \tilde{y}_{\psi}^{k}}}{y_{\psi}^{i} \tilde{y}_{\psi}^{j}\left(Y^{u^{\dagger}} Y^{u}\right)_{i j}} .
\end{gathered}
$$

The 1-loop $\Phi_{N} \rightarrow \Phi_{N}$ diagram gives the first term whereas the $\Phi_{N}^{2} H^{\dagger} H$ box diagram gives the second term. For the loop diagram generating the first term, we have taken the cutoff for the $\psi u^{c}$ loop to be the mass of the clockwork radial modes, $m_{\rho} \sim f$.

\section{B. Nelson-Barr solution to the strong $\boldsymbol{C P}$ problem}

The Lagrangian in Eq. (16) also provides a solution to the strong $C P$ problem. Once the relaxion stops, the phase, $\theta_{N}=\left\langle\pi_{N}\right\rangle / f \sim \phi / F$, enters the $4 \times 4$ matrix for the up sector,

$$
M_{u}=\left(\begin{array}{cc}
\left(\mu_{\psi}\right)_{1 \times 1} & (B)_{1 \times 3} \\
(0)_{3 \times 1} & \left(v Y^{u}\right)_{3 \times 3}
\end{array}\right),
$$

where $B_{i}=f\left(y_{\psi}^{i} e^{i \theta_{N}}+\tilde{y}_{\psi}^{i} e^{-i \theta_{N}}\right) / \sqrt{2}$. The phase, $\theta_{N}$, is nothing but the phase of the cosine of the rolling potential at the relaxion stopping point. Note that the bottom-left element in the above mass matrix is zero due to the absence of the $Q H \psi^{c}$ term in the Lagrangian, which in turn is a direct consequence of the $Z_{2}$ symmetry. This ensures that at tree level there is no contribution to $\bar{\theta}_{\mathrm{QCD}}$ from the phase, $\theta_{N}$, as $\operatorname{Arg}\left(\operatorname{det}\left(M_{u}\right)\right)=0$, where we use the fact that $\mu_{\psi}$ is real. On the other hand, for $\mu^{2}+B_{i} B_{i}^{*} \gg v^{2}$, we can integrate out the vectorlike pair to give an effective $3 \times 3$ mass squared matrix of the SM up quark sector with an $\mathcal{O}(1)$ phase. This gets translated into an $\mathcal{O}(1)$ phase in the CKM matrix $V_{\mathrm{CKM}}=V_{u_{L}}^{\dagger} V_{d_{L}}$ (see [11]). Radiative effects can spoil the solution to the strong $C P$ problem unless $y_{\psi} \lesssim 10^{-2}[11]$.

Before going to the next section we would like to comment that there appears to be no obvious difficulty in extending our model along the lines of [12] to also address the SM flavor puzzle for the charged leptons and quarks. At the cost of complicating our model, this can be achieved by identifying one of the intermediate sites of the clockwork chain with the flavon for the charged fermions and the Abelian symmetry at this site with a FroggattNielsen flavor symmetry. In order not to generate a $\bar{\theta}_{\mathrm{QCD}}$, the charge assignment of the SM fermions must be anomalyfree with respect to QCD as emphasised in [12] where an example charge assignment was also presented. We do not explore this direction further and stick to our more minimal setup here.

\section{PARAMETER SPACE}

First, let us consider the constraints that arise on the SRB scenario for the right-handed neutrino current introduced in Sec. III. We fix the $f_{k} / f$ ratio according to Eq. (15) such that each point in the plot gives the correct baryon asymmetry. The vertical green band shows the region that is ruled out by requiring, $m_{\phi}<5 H\left(T_{c}\right)$, the condition in Eq. (5) that the relaxion does not overshoot the barriers of the backreaction potential once they reappear after reheating. Here we have taken the maximal value $T_{c}=T_{\text {sph }}$ (see Sec. III). The blue shaded region corresponds to the region $\Lambda_{\mathrm{c}}^{2}>16 \pi^{2} v^{2}$ ruled out by the requirement (derived in [7]) that the Higgs-dependent parts of the backreaction potential dominate over any Higgs-independent contribution. The dashed lines show the required value of $\tan \left(\phi_{0} / f_{k}\right)$ to reproduce the correct dark matter density in Eq. (7). As explained below Eq. (7), the extent to which $\tan \frac{\phi_{0}}{f}$ deviates from unity can be interpreted as a measure of the required tuning. The orange region shows fifth force constraints that arise due to the fact that the relaxion mixes with the Higgs boson with a mixing angle (see [32]), $\sin \theta \sim \frac{\Lambda_{c}^{4}}{f_{k} v m_{h}^{2}}$.

The rest of the constraints arise from the implementation of the Nelson-Barr mechanism in Sec. IV. First of all, from Eq. (18) we see that for a given value of $f$ and $y_{\psi}$ one can fix the value of the Higgs mass cutoff, $M$, giving us the scale on the right-hand side of the frame. The red band at the top shows the region where the value of the cutoff, $M$, exceeds the upper bound imposed in Eq. (4). The grey band 


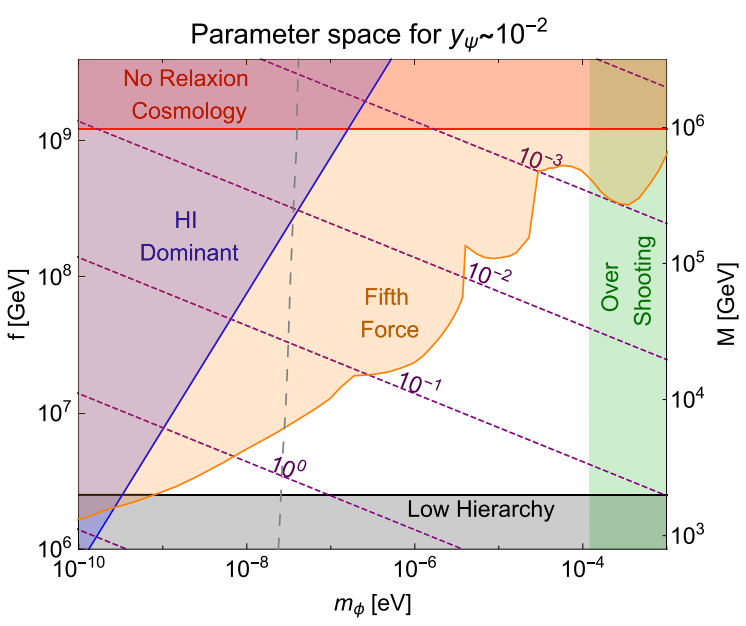

FIG. 2. The parameter space for an all-in-one relaxion in the $\left(m_{\phi}, f\right)$ plane. For each value of $f$, taking the maximal value $y_{\psi}=10^{-2}$, the value of the Higgs mass cut-off, $M$, is fixed as shown on the right-hand side of the frame [see Eq. (17)]. The red band shows the region violating the bounds imposed by cosmological requirements in [7]; blue denotes the region where the Higgs-independent contributions to the backreaction are no longer subdominant [7]; the orange region shows the fifth-force exclusions due to mixing of $\phi$ with the Higgs [32]; green denotes the region in which the relaxion overshoots the backreaction barriers after reheating (see Sec. II) for the maximal value $T_{c}=$ $T_{\text {sph }}$ and the purple dashed lines show the tuning needed to reproduce the correct relic density [8]. The grey band at the bottom shows the region where the relaxion mechanism is unable to raise the Higgs cut-off beyond $2 \mathrm{TeV}$. Finally, the region to the left of the dashed line may be probed in future atomic physics experiments if there is an overdensity of relaxion dark matter around the Earth [33,34].

at the bottom shows the region where the relaxion mechanism is unable to raise the Higgs cutoff beyond $2 \mathrm{TeV}$. Finally, the region to the left of the dashed line may be probed in future atomic physics experiments if there is an overdensity of relaxion dark matter around the earth [33,34]. We see from Fig. 2 that after all the constraints are imposed, a finite allowed region remains that remarkably contains the region in which tuning to obtain the correct relic density for dark matter is minimal.

We will comment in the next section on how this allowed region can be probed further by future experiments.

\section{DISCUSSION AND CONCLUSION}

We have presented a simultaneous solution to five BSM puzzles: namely the lightness of the Higgs boson in the absence of $\mathrm{TeV}$ scale new physics, dark matter, matterantimatter asymmetry, neutrino masses and the strong $C P$ problem. While our construction is admittedly more involved than some other attempts to solve BSM puzzles in a unified way $[1,2,5,6]$, this is because we also use cosmological relaxation to achieve the challenging task of obtaining a light Higgs boson without adding any $\mathrm{TeV}$ scale states that cutoff the Higgs mass divergence. Our construction has all the ingredients of a standard relaxion model - such as a chain of clockwork scalars and a TeVscale strong sector-but beyond this we only make minimal modifications by adding three RH neutrinos and an up-type $\mathrm{SU}(2)_{L}$ singlet vectorlike quark pair.

Our all-in-one relaxion setup gives a diverse set of observational predictions. Our construction predicts the absence of a $C P$-violating phase in the neutrino mass matrix and $\mathrm{GeV}$-scale sterile neutrinos potentially close to the reach of future experiments such as SHiP [29]. The strong $C P$ phase in our model is nonzero and may be detectable in future experiments. The finite allowed parameter space in Fig. 2 can also be probed by future atomic physics experiments $[33,34]$ or future improvements in fifth force experiments. Finally, we would like to point out the interesting trade-off that exists in Fig. 2 between the Higgs mass cutoff scale and the tuning required to reproduce the correct abundance of dark matter. The least tuned regions correspond to cutoff values smaller than $100 \mathrm{TeV}$, a scale where top partners in a full solution to the hierarchy problem can be seen in future high energy colliders.

We conclude by mentioning an interesting future direction. Our construction involves the standard relaxion mechanism, which utilises Hubble friction to stop the relaxion. It will be interesting to see if some of our ideas can be implemented in alternative models involving particle production [35-38], which are attractive because they decouple the relaxion mechanism from inflation.

\section{ACKNOWLEDGMENTS}

We would like to thank S. Abel, J. Scholtz and A. Titov for very useful discussions.
[1] G. Ballesteros, J. Redondo, A. Ringwald, and C. Tamarit, Unifying Inflation with the Axion, Dark Matter, Baryogenesis and the Seesaw Mechanism, Phys. Rev. Lett. 118, 071802 (2017).
[2] G. Ballesteros, J. Redondo, A. Ringwald, and C. Tamarit, Standard Model-axion-seesaw-Higgs portal inflation. Five problems of particle physics and cosmology solved in one stroke, J. Cosmol. Astropart. Phys. 08 (2017) 001. 
[3] A. Salvio, A simple motivated completion of the Standard Model below the Planck scale: Axions and right-handed neutrinos, Phys. Lett. B 743, 428 (2015).

[4] A. Salvio, Critical Higgs inflation in a viable motivated model, Phys. Rev. D 99, 015037 (2019).

[5] Y. Ema, K. Hamaguchi, T. Moroi, and K. Nakayama, Flaxion: A minimal extension to solve puzzles in the standard model, J. High Energy Phys. 01 (2017) 096.

[6] L. Calibbi, F. Goertz, D. Redigolo, R. Ziegler, and J. Zupan, Minimal axion model from flavor, Phys. Rev. D 95, 095009 (2017).

[7] P. W. Graham, D. E. Kaplan, and S. Rajendran, Cosmological Relaxation of the Electroweak Scale, Phys. Rev. Lett. 115, 221801 (2015).

[8] S. A. Abel, R. S. Gupta, and J. Scholtz, Out-of-the-box baryogenesis during relaxation, Phys. Rev. D 100, 015034 (2019).

[9] A. E. Nelson, Naturally weak $C P$ violation, Phys. Lett. 136B, 387 (1984).

[10] S. M. Barr, Solving the Strong CP Problem without the Peccei-Quinn Symmetry, Phys. Rev. Lett. 53, 329 (1984).

[11] O. Davidi, R. S. Gupta, G. Perez, D. Redigolo, and A. Shalit, The Nelson-Barr relaxion, Phys. Rev. D 99, 035014 (2019).

[12] O. Davidi, R. S. Gupta, G. Perez, D. Redigolo, and A. Shalit, The hierarchion, a relaxion addressing the Standard Model's hierarchies, J. High Energy Phys. 08 (2018) 153.

[13] A. Banerjee, H. Kim, and G. Perez, Coherent relaxion dark matter, arXiv:1810.01889.

[14] C. D. Froggatt and H.B. Nielsen, Hierarchy of quark masses, Cabibbo angles and $C P$ violation, Nucl. Phys. B147, 277 (1979).

[15] The periodicity $F$ is large such that the cosine is locally linear in $\phi$. By expanding the cosine terms about an arbitrary point, the polynomial terms of the original work [7] can be recovered with $g \sim M^{2} / F$.

[16] B. Batell, G. F. Giudice, and M. McCullough, Natural heavy supersymmetry, J. High Energy Phys. 12 (2015) 1.

[17] B. Batell, M. A. Fedderke, and L.-T. Wang, Relaxation of the composite Higgs little hierarchy, J. High Energy Phys. 12 (2017) 139.

[18] M. Kawasaki, T. Kobayashi, and F. Takahashi, NonGaussianity from curvatons revisited, Phys. Rev. D 84, 123506 (2011).

[19] K. Choi, H. Kim, and T. Sekiguchi, Dynamics of the cosmological relaxation after reheating, Phys. Rev. D 95, 075008 (2017).
[20] R. S. Gupta, Relaxion measure problem, Phys. Rev. D 98, 055023 (2018).

[21] R. S. Gupta, Z. Komargodski, G. Perez, and L. Ubaldi, Is the relaxion an axion?, J. High Energy Phys. 02 (2016) 166.

[22] K. Choi, H. Kim, and S. Yun, Natural inflation with multiple sub-Planckian axions, Phys. Rev. D 90, 023545 (2014).

[23] K. Choi and S. H. Im, Realizing the relaxion from multiple axions and its UV completion with high scale supersymmetry, J. High Energy Phys. 01 (2016) 149.

[24] D. E. Kaplan and R. Rattazzi, Large field excursions and approximate discrete symmetries from a clockwork axion, Phys. Rev. D 93, 085007 (2016).

[25] A. G. Cohen and D. B. Kaplan, Thermodynamic generation of the Baryon asymmetry, Phys. Lett. B 199, 251 (1987).

[26] A. G. Cohen and D. B. Kaplan, Spontaneous Baryogenesis, Nucl. Phys. B308, 913 (1988).

[27] M. D’Onofrio, K. Rummukainen, and A. Tranberg, Sphaleron Rate in the Minimal Standard Model, Phys. Rev. Lett. 113, 141602 (2014).

[28] K. Dick, M. Lindner, M. Ratz, and D. Wright, Leptogenesis with Dirac Neutrinos, Phys. Rev. Lett. 84, 4039 (2000).

[29] S. Alekhin et al., A facility to search for hidden particles at the CERN SPS: The SHiP physics case, Rep. Prog. Phys. 79, 124201 (2016).

[30] F. Capozzi, E. Lisi, A. Marrone, and A. Palazzo, Current unknowns in the three neutrino framework, Prog. Part. Nucl. Phys. 102, 48 (2018).

[31] L. Bento, G. C. Branco, and P. A. Parada, A minimal model with natural suppression of strong $C P$ violation, Phys. Lett. B 267, 95 (1991).

[32] T. Flacke, C. Frugiuele, E. Fuchs, R. S. Gupta, and G. Perez, Phenomenology of relaxion-Higgs mixing, J. High Energy Phys. 06 (2017) 050.

[33] S. Aharony, N. Akerman, R. Ozeri, G. Perez, I. Savoray, and R. Shaniv, Constraining rapidly oscillating scalar dark matter using dynamic decoupling, arXiv:1902.02788.

[34] A. Banerjee, D. Budker, J. Eby, H. Kim, and G. Perez, Relaxion stars and their detection via atomic physics, arXiv:1902.08212.

[35] A. Hook and G. Marques-Tavares, Relaxation from particle production, J. High Energy Phys. 12 (2016) 101.

[36] N. Fonseca, E. Morgante, and G. Servant, Higgs relaxation after inflation, J. High Energy Phys. 10 (2018) 020.

[37] M. Son, F. Ye, and T. You, Leptogenesis in cosmological relaxation with particle production, Phys. Rev. D 99, 095016 (2019).

[38] N. Fonseca and E. Morgante, Relaxion dark matter, arXiv: 1809.04534. 